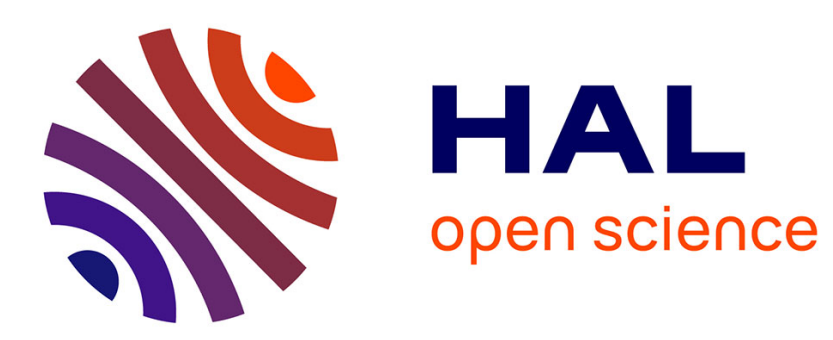

\title{
New characterization of the kernel of the n-dimensional Laplace operator in exterior domains
}

\author{
Chérif Amrouche, Huy Hoang Nguyen
}

\section{To cite this version:}

Chérif Amrouche, Huy Hoang Nguyen. New characterization of the kernel of the n-dimensional Laplace operator in exterior domains. 2008. hal-00297271

\section{HAL Id: hal-00297271 \\ https://hal.science/hal-00297271}

Preprint submitted on 15 Jul 2008

HAL is a multi-disciplinary open access archive for the deposit and dissemination of scientific research documents, whether they are published or not. The documents may come from teaching and research institutions in France or abroad, or from public or private research centers.
L'archive ouverte pluridisciplinaire HAL, est destinée au dépôt et à la diffusion de documents scientifiques de niveau recherche, publiés ou non, émanant des établissements d'enseignement et de recherche français ou étrangers, des laboratoires publics ou privés. 


\title{
New characterization of the kernel of the $n$-dimensional Laplace operator in exterior domains
}

\author{
Chérif AMROUCHE* and Huy Hoang NGUYEN ${ }^{\dagger}$ \\ Laboratoire de Mathématiques Appliquées \\ CNRS UMR 5142 \\ Université de Pau et des Pays de l'Adour \\ IPRA - Avenue de l'Université 64013 Pau, France \\ *cherif.amrouche@univ-pau.fr \\ ${ }_{\dagger}^{\dagger}$ huy-hoang.nguyen@etud.univ-pau.fr
}

\begin{abstract}
In this Note, we study the characterization of the kernel of the Laplace operator with Dirichlet boundary conditions in exterior domains. We consider data in weighted Sobolev spaces.

Résumé

Nouvelle caractérisation du noyau du laplacien en domaine extérieur. Nous étudions dans cet article la caractérisation du noyau de l'opérateur laplacien avec des conditions de Dirichlet au bord dans un ouvert extérieur. Nous considérons des données dans des espaces de Sobolev avec poids.
\end{abstract}

\section{Introduction}

Let $\Omega^{\prime}$ be a bounded open region of $\mathbb{R}^{n}(n \geq 2)$, not necessarily connected, with a Lipschitz-continuous boundary $\Gamma$ and let $\Omega$ be the complement of $\overline{\Omega^{\prime}}$. We suppose that $\Omega^{\prime}$ has a finite number of connected components and each connected component has a connected boundary, so that $\Omega$ is connected. The purpose of this Note is to characterize the kernel $\mathcal{A}^{p, q}(\Omega)$ of the Laplace operator with Dirichlet boundary conditions:

$$
\mathcal{A}^{p, q}(\Omega)=\left\{z \in W_{0}^{1, p}(\Omega)+W_{0}^{1, q}(\Omega) ; \Delta z=0 \text { in } \Omega \text { and } z=0 \text { on } \Gamma\right\} .
$$

The reason why we suggest an idea to study $\mathcal{A}^{p, q}(\Omega)$ is explained in Remark 2.2 . Since the problem is posed in a $n$-dimensional exterior domain, it is important to specify the behavior at infinity for the data and solutions. We have chosen to impose such conditions by setting our problem in weighted Sobolev spaces which provide a correct functional setting for unbounded domains. It means that the growth and decay of functions at infinity are expressed by means of weights, in particular, the function in these weighted Sobolev spaces satisfies an optimal weighted Poincaré-type inequality. In the whole text, bold characters are used for vector or matrix fields. We now introduce the definition of weighted 
Sobolev spaces and some its properties. A typical point in $\mathbb{R}^{n}$ is denoted by $\boldsymbol{x}=\left(x_{1}, \ldots, x_{n}\right)$ and its norm is given by $r=|\boldsymbol{x}|=\left(x_{1}^{2}+\ldots+x_{n}^{2}\right)^{\frac{1}{2}}$. We define the weight function $\rho(\boldsymbol{x})=1+r$. For each $p \in \mathbb{R}$ and $1<p<\infty$, the conjugate exponent $p^{\prime}$ is given by the relation $\frac{1}{p}+\frac{1}{p^{\prime}}=1$. We now define the weighted Sobolev space

$$
W_{0}^{1, p}(\Omega)=\left\{u \in \mathcal{D}^{\prime}(\Omega), \frac{u}{w} \in L^{p}(\Omega), \nabla u \in \mathbf{L}^{p}(\Omega)\right\}
$$

where

$$
w=\left\{\begin{array}{l}
(1+r) \quad \text { if } p \neq n, \\
(1+r) \ln (2+r) \quad \text { if } p=n .
\end{array}\right.
$$

This space is a reflexive Banach space when endowed with the norm:

$$
\|u\|_{W_{0}^{1, p}(\Omega)}=\left(\left\|\frac{u}{w}\right\|_{L^{p}(\Omega)}^{p}+\|\nabla u\|_{\mathbf{L}^{p}(\Omega)}^{p}\right)^{1 / p} .
$$

We note that the logarithmic weight only appears if $p=n$ and all the local properties of $W_{0}^{1, p}(\Omega)$ coincide with those of the corresponding classical Sobolev space $W^{1, p}(\Omega)$. We set $\stackrel{\circ}{W}_{0}^{1, p}(\Omega)=\overline{\mathcal{D}(\Omega)} W_{0}^{1, p}(\Omega)$ and we denote the dual space of $\stackrel{\circ}{W}_{0}^{1, p}(\Omega)$ by $W_{0}^{-1, p^{\prime}}(\Omega)$, which is a space of distributions. When $\Omega=\mathbb{R}^{n}$, we have $W_{0}^{1, p}\left(\mathbb{R}^{n}\right)=\stackrel{\circ}{W}_{0}^{1, p}\left(\mathbb{R}^{n}\right)$. We have the algebraic and topological imbeddings

$$
W_{0}^{1, p}(\Omega) \hookrightarrow W_{-1}^{0, p}(\Omega) \text { if } p \neq n,
$$

where

$$
W_{-1}^{0, p}(\Omega)=\left\{u \in \mathcal{D}^{\prime}(\Omega), \frac{u}{1+r} \in L^{p}(\Omega)\right\} .
$$

For all $\lambda \in \mathbb{N}^{n}$ where $0 \leq|\lambda| \leq 2$, the mapping

$$
u \in W_{0}^{1, p}(\Omega) \rightarrow \partial^{\lambda} u \in W_{0}^{1-|\lambda|, p}(\Omega)
$$

is continuous. Also recall the following Sobolev embeddings (see [1]):

$$
W_{0}^{1, p}(\Omega) \hookrightarrow L^{p^{*}}(\Omega) \text { where } p^{*}=\frac{n p}{n-p} \text { and } 1<p<n .
$$

Note that $\mathbb{R} \subset W_{0}^{1, p}(\Omega)$ if and only if $p \geq n$. We now set that

$$
\mathcal{A}^{p}(\Omega)=\left\{y \in W_{0}^{1, p}(\Omega) ; \Delta y=0 \text { in } \Omega \text { and } y=0 \text { on } \Gamma\right\} .
$$

In the two-dimensional space, let $U=\frac{1}{2 \pi} \ln r$ be the fundamental solution of Laplace's equation. We now define

$$
u_{0}=U *\left(\frac{1}{|\Gamma|} \delta_{\Gamma}\right)
$$

where $\delta_{\Gamma}$ is the distribution defined by

$$
\forall \varphi \in \mathcal{D}\left(\mathbb{R}^{2}\right), \quad<\delta_{\Gamma}, \varphi>=\int_{\Gamma} \varphi d \sigma .
$$

The next lemma characterizes the kernel $A^{p}(\Omega)$ (see [3]). 
Lemma 1.1. Let $1<p<\infty$ and suppose that $\Gamma$ is of class $C^{1,1}$.

i) If $p<n$ or if $p=n=2$, then $\mathcal{A}^{p}(\Omega)=\{0\}$.

ii) If $p \geq n \geq 3$, then

$$
\mathcal{A}^{p}(\Omega)=\{c(\lambda-1) ; c \in \mathbb{R}\},
$$

where $\lambda \in \bigcap_{r>\frac{n}{n-1}} W_{0}^{1, r}(\Omega)$ is the unique solution of the following problem

$$
\Delta \lambda=0 \text { in } \Omega \text { and } \lambda=1 \text { on } \Gamma .
$$

iii) If $p>n=2$, then

$$
\mathcal{A}^{p}(\Omega)=\left\{c\left(\mu-u_{0}\right) ; c \in \mathbb{R}\right\},
$$

where $u_{0}$ is defined by (1.2) and $\mu$ is the only solution in $\bigcap_{r>2} W_{0}^{1, r}(\Omega)$ of the problem

$$
\Delta \mu=0 \text { in } \Omega \text { and } \mu=u_{0} \text { on } \Gamma \text {. }
$$

Remark 1.2. When $\Gamma$ is the unit sphere of $\mathbb{R}^{n}(n \geq 3)$, then $\lambda=\frac{1}{|\boldsymbol{x}|^{n-2}}$. Note that $\nabla \lambda \in \mathbf{L}^{n /(n-1), \infty}\left(\mathbb{R}^{n}\right)$ and $\frac{\lambda}{w} \in L^{n /(n-1), \infty}\left(\mathbb{R}^{n}\right)$, where the weak-type space $L^{p, \infty}\left(\mathbb{R}^{n}\right)$ is defined as follows

$$
u \in L^{p, \infty}\left(\mathbb{R}^{n}\right) \Leftrightarrow \sup _{t>0} t\left(\int_{\left\{\boldsymbol{x} \in \mathbb{R}^{n},|u(\boldsymbol{x})|>t\right\}} d \boldsymbol{x}\right)^{1 / p}<\infty .
$$

Then we will write $\lambda \in W_{0, \infty}^{1, n /(n-1)}\left(\mathbb{R}^{n}\right)$.

\section{Main results}

In this section, we give a theorem that characterizes the kernel $\mathcal{A}^{p, q}(\Omega)$ of the Laplace operator with Dirichlet boundary conditions:

$$
\mathcal{A}^{p, q}(\Omega)=\left\{z \in W_{0}^{1, p}(\Omega)+W_{0}^{1, q}(\Omega) ; \Delta z=0 \text { in } \Omega \text { and } z=0 \text { on } \Gamma\right\},
$$

with $1<p<q<\infty$.

Theorem 2.1. Let $1<p<q<\infty$ and $\Omega \subset \mathbb{R}^{n}$ be an exterior domain with $C^{1,1}$ boundary.

i) If $q<n$ or if $q=n=2$, then $\mathcal{A}^{p, q}(\Omega)=\{0\}$.

ii) If $q \geq n \geq 3$, then

$$
\mathcal{A}^{p, q}(\Omega)=\{c(\lambda-1) ; c \in \mathbb{R}\}
$$

where $\lambda \in \bigcap_{r>\frac{n}{n-1}} W_{0}^{1, r}(\Omega)$ is the unique solution of the problem (1.3).

iii) If $q>n=2$, then

$$
\mathcal{A}^{p, q}(\Omega)=\left\{c\left(\mu-u_{0}\right) ; c \in \mathbb{R}\right\}
$$

where $\mu \in \bigcap_{r>2} W_{0}^{1, r}(\Omega)$ is the unique solution of the problem (1.4). 
Proof. Let $z \in \mathcal{A}^{p, q}(\Omega)$, then $z=u-v$ with $u \in W_{0}^{1, p}(\Omega), v \in W_{0}^{1, q}(\Omega)$ and $u=v$ on $\Gamma$. Let now $\widetilde{v} \in W_{0}^{1, q}\left(\mathbb{R}^{n}\right)$ an extended function of $v$ outside $\Omega$. We set $\widetilde{u}=u$ in $\Omega, \widetilde{u}=\widetilde{v}$ outside $\Omega$ and $\widetilde{z}=\widetilde{u}-\widetilde{v}$. It is easy to see that $\widetilde{z}$ in $W_{0}^{1, p}\left(\mathbb{R}^{n}\right)+W_{0}^{1, q}\left(\mathbb{R}^{n}\right)$ and $\widetilde{z}=0$ outside $\Omega$. Set now $h=\Delta \widetilde{z}$. As supp $h \subset \Gamma$, then $h \in W_{0}^{-1, p}\left(\mathbb{R}^{n}\right)$.

A. If $n \geq 3$ : We consider 3 following cases:

1) The case $\frac{n}{n-1}<p$ : We know that there exists $w \in W_{0}^{1, p}\left(\mathbb{R}^{n}\right)$ such that $\Delta w=h$ in $\mathbb{R}^{n}$. The difference $w-\widetilde{z}$ belongs to $W_{0}^{1, p}\left(\mathbb{R}^{n}\right)+W_{0}^{1, q}\left(\mathbb{R}^{n}\right)$ and is harmonic in $\mathbb{R}^{n}$. We begin by supposing that $q<n$. We deduce that $w=\widetilde{z}$ in $\mathbb{R}^{n}$ and then $w$ vanishes on $\Gamma$. Since $p<n$, thanks to Lemma 2.10 [3], $w$ is unique and $w=0$ in $\Omega$, i.e., $z=0$ in $\Omega$. Now if $q \geq n$, there exists a constant $c$ such that $w-\widetilde{z}=c$ and $w=c$ on $\Gamma$. If $p<n$, from Lemma 2.10 [3], then $w$ is unique and $w=c \lambda$ in $\Omega$ where $\lambda \in \bigcap_{r>\frac{n}{n-1}} W_{0}^{1, r}(\Omega)$ is a unique solution of the system (1.3). Therefore, we can deduce $z=c(\lambda-1)$ in $\Omega$. If $p \geq n$, it is easy to deduce that $w$ is unique up to a constant and we still obtain that $z=c(\lambda-1)$ in $\Omega$.

2) The case $1<p<\frac{n}{n-1}$ : In the n-dimensional case, let $E(\boldsymbol{x})=c_{n}|\boldsymbol{x}|^{2-n}$ be the fundamental solution of Laplace's equation. As $\delta \in W_{0}^{-1, p}\left(\mathbb{R}^{n}\right)$ is the Dirac distribution, then there exists a unique $w_{0} \in W_{0}^{1, p}\left(\mathbb{R}^{n}\right)$ such that

$$
\Delta w_{0}=h-\delta<h, 1>_{W_{0}^{-1, p}\left(\mathbb{R}^{n}\right) \times W_{0}^{1, p^{\prime}}\left(\mathbb{R}^{n}\right)} \text { in } \mathbb{R}^{n} .
$$

We now set that

$$
w=w_{0}-E<h, 1>_{W_{0}^{-1, p}\left(\mathbb{R}^{n}\right) \times W_{0}^{1, p^{\prime}}\left(\mathbb{R}^{n}\right)} .
$$

Then $\Delta w=h$ in $\mathbb{R}^{n}$ and $w-\widetilde{z}$ is harmonic. Note that the origin is not in $\Omega$. The restriction of $w$ to $\Omega$ belongs to $W_{0}^{1, p}(\Omega)+W_{0}^{1, r}(\Omega)$ for all $r>\frac{n}{n-1}$. The function $w$ belongs to $W_{0}^{1, p}\left(\mathbb{R}^{n}\right)+W_{0, \infty}^{1, n /(n-1)}\left(\mathbb{R}^{n}\right)$, i.e., $\nabla w \in \mathbf{L}^{p}\left(\mathbb{R}^{n}\right)+\mathbf{L}^{n /(n-1), \infty}\left(\mathbb{R}^{n}\right)$. Hence, the difference $w-\widetilde{z}$ belongs to $W_{0}^{1, p}\left(\mathbb{R}^{n}\right)+W_{0, \infty}^{1, n /(n-1)}\left(\mathbb{R}^{n}\right)+W_{0}^{1, q}\left(\mathbb{R}^{n}\right)$. a) The case $q<n$ : We deduce $w=\widetilde{z}$ in $\mathbb{R}^{n}$ and $w=0$ on $\Gamma$. Then $\Delta w_{0}=0$ in $\Omega$ and $w_{0}=<h, 1>E$ on $\Gamma$. As $p^{\prime}>n$, for any $\varphi \in \mathcal{A}^{p^{\prime}}(\Omega)$ and for any $\psi \in \mathcal{D}(\bar{\Omega})$, we have the following Green's formula

$$
\int_{\Omega} \psi \Delta \varphi d \boldsymbol{x}=\int_{\Omega} \varphi \Delta \psi d \boldsymbol{x}+\left\langle\frac{\partial \varphi}{\partial \boldsymbol{n}}, \psi\right\rangle_{\Gamma}-\left\langle\varphi, \frac{\partial \psi}{\partial \boldsymbol{n}}\right\rangle_{\Gamma}
$$

where $<., .>_{\Gamma}$ denotes the duality between $W^{\frac{-1}{p}, p^{\prime}}(\Gamma)$ and $W^{1-\frac{1}{p}, p}(\Gamma)$. Then, we deduce that

$$
\int_{\Omega} \varphi \Delta \psi d \boldsymbol{x}=-\left\langle\frac{\partial \varphi}{\partial \boldsymbol{n}}, \psi\right\rangle_{\Gamma}
$$

Thanks to the density of $\mathcal{D}(\bar{\Omega})$ in $W_{0}^{1, p}(\Omega)$, for all $\varphi \in \mathcal{A}^{p^{\prime}}(\Omega)$ and for all $v \in W_{0}^{1, p}(\Omega)$, we have

$$
<\Delta v, \varphi>_{W_{0}^{-1, p}(\Omega) \times \stackrel{\circ}{W}_{0}^{1, p^{\prime}}(\Omega)}=-<\frac{\partial \varphi}{\partial \boldsymbol{n}}, v>_{W^{\frac{-1}{p}, p^{\prime}}(\Gamma) \times W^{1-\frac{1}{p}, p}(\Gamma)} .
$$


Applying (2.1) with $v=w_{0} \in W_{0}^{1, p}(\Omega)$ and $\varphi=\lambda-1 \in \mathcal{A}^{p^{\prime}}(\Omega)$, we obtain

$$
<h, 1><E, \frac{\partial \lambda}{\partial \boldsymbol{n}}>_{W^{1-\frac{1}{p}, p}(\Gamma) \times W^{\frac{-1}{p}, p^{\prime}}(\Gamma)}=0 .
$$

Note that,

$$
<E, \frac{\partial \lambda}{\partial \boldsymbol{n}}>_{\Gamma}=<\frac{\partial E}{\partial \boldsymbol{n}}, \lambda>_{\Gamma}=\int_{\Gamma} \frac{\partial E}{\partial \boldsymbol{n}} .
$$

Let $B_{R}$ the open ball of radius $R>0$ centered at the origin such that $\Omega^{\prime} \subset B_{R}$ and set that $\Omega_{R}=\Omega \cap B_{R}$. Then we have

$$
0=\int_{\Omega_{R}} \Delta E=\int_{\Gamma} \frac{\partial E}{\partial \boldsymbol{n}}-\int_{\partial B_{R}} \frac{\partial E}{\partial \boldsymbol{n}}
$$

It is easy to verify that $\int_{\partial B_{R}} \frac{\partial E}{\partial \boldsymbol{n}}=1$, then $\langle h, 1>=0$. Consequently, from Lemma 2.10 [3], we deduce $w_{0}=0$ in $\Omega$. Therefore, $w=0$ and $z=0$ in $\Omega$.

b) The case $q \geq n$ : There exists a constant $c$ such that $w-\widetilde{z}=c$ in $\mathbb{R}^{n}$ and $w=c$ on $\Gamma$. Then, $\Delta w_{0}=0$ in $\Omega$ and $w_{0}=c+<h, 1>E$ on $\Gamma$. Applying again (2.1), we obtain

$$
<c+<h, 1>E, \frac{\partial \lambda}{\partial \boldsymbol{n}}>_{\Gamma}=0 .
$$

Set that $\mu=c+<h, 1>E$ on $\Gamma$. It is not difficult to see that $\mu \in W^{1-\frac{1}{r}, r}(\Gamma)$ with any $r \in] \frac{n}{n-1}, n\left[\right.$. Then there exists a unique $y \in W_{0}^{1, r}(\Omega)$ such that $\Delta y=0$ in $\Omega$ and $y=\mu$ on $\Gamma$. Then, we deduce that $y-w_{0} \in \mathcal{A}^{p, r}(\Omega)$. Thanks to the results for the case $2 a$ ) of this Lemma, we have $y=w_{0}$, i.e., $w_{0} \in W_{0}^{1, p}(\Omega) \cap W_{0}^{1, r}(\Omega)$. We can see that $\mu$ also belongs to $W^{1-\frac{1}{q}, q}(\Gamma)$. Then there exists $\theta \in W_{0}^{1, q}(\Omega)$ such that $\Delta \theta=0$ in $\Omega$ and $\theta=\mu$ on $\Gamma$. Then, $\theta-w_{0} \in \mathcal{A}^{r, q}(\Omega)$. From the case 1$)$, there exists a constant $\alpha$ such that $\theta-w_{0}=\alpha(\lambda-1)$ and we deduce that $w_{0} \in W_{0}^{1, q}(\Omega)$. Consequently, the function $w \in W_{0}^{1, q}(\Omega)$ and since $w=c$ on $\Gamma$ and from the characterization of $\mathcal{A}^{q}(\Omega)$, we can immediately deduce that $w=c \lambda$ and $z=c(\lambda-1)$ in $\Omega$.

3) The case $p=\frac{n}{n-1}$ : Finally, let $\varphi \in \mathcal{D}\left(\mathbb{R}^{n}\right)$ satisfying $\int_{\mathbb{R}^{n}} \varphi=1$ and $\mu=E * \varphi$. We know that $\mu \in L^{n, \infty}\left(\mathbb{R}^{n}\right) \cap L^{r}\left(\mathbb{R}^{n}\right)$ for any $r>n$ and $\nabla \mu \in L^{n /(n-1), \infty}\left(\mathbb{R}^{n}\right) \cap L^{s}\left(\mathbb{R}^{n}\right)$ for any $s>\frac{n}{n-1}$. The reasonning applies by remplacing $\delta$ by $\varphi$ and $E$ by $\mu$.

B. If $n=2$ : We know that there exists a unique $w_{0} \in W_{0}^{1, p}\left(\mathbb{R}^{2}\right)$ satisfying

$$
\Delta w_{0}=h-<h, 1>_{W_{0}^{-1, p}\left(\mathbb{R}^{2}\right) \times W_{0}^{1, p^{\prime}}\left(\mathbb{R}^{2}\right)} \Delta u_{0} \text { in } \mathbb{R}^{2},
$$

where $u_{0}$ is defined by (1.2). Now we set

$$
w=w_{0}+<h, 1>_{W_{0}^{-1, p}\left(\mathbb{R}^{2}\right) \times W_{0}^{1, p^{\prime}}\left(\mathbb{R}^{2}\right)} u_{0} .
$$

Then $\Delta w=h$ in $\mathbb{R}^{2}$ and $w-\widetilde{z}$ is harmonic. Proceeding as in the case A2 by distinguishing 2 cases $q \leq 2$ and $q>2$, we obtain results and the proof is finished. 
Remark 2.2. Let $f \in W_{0}^{-1, p}(\Omega), g \in W^{1-\frac{1}{p}, p}(\Gamma)$ and $u \in W_{0}^{1, p}(\Omega)$ be a solution of the following system

$$
-\Delta u=f \text { in } \Omega \text { and } u=g \text { on } \Gamma .
$$

We recall that the existence of this solution $u$ if and only if $f$ and $g$ satisfy the compatibility condition

$$
\forall \varphi \in \mathcal{A}^{p^{\prime}}(\Omega), \quad\langle f, \varphi\rangle_{W_{0}^{-1, p}(\Omega) \times \stackrel{\circ}{W}_{0}^{1, p^{\prime}}(\Omega)}=\left\langle g, \frac{\partial \varphi}{\partial \boldsymbol{n}}\right\rangle_{W^{1-\frac{1}{p}, p}(\Gamma) \times W^{\frac{-1}{p}, p^{\prime}}(\Gamma)} .
$$

If, in addition, $f \in W_{0}^{-1, q}(\Omega), g \in W^{1-\frac{1}{q}, q}(\Gamma)$ with $p<q$ satisfying the compatibility condition (2.2) by remplacing $p$ by $q$, the question "Does the solution $u$ belong to $W_{0}^{1, q}(\Omega)$ ?" is risen. Since there exists $v \in W_{0}^{1, q}(\Omega)$ satisfying $-\Delta v=f$ in $\Omega$ and $v=g$ on $\Gamma$ and from Theorem 2.1, we obtain $u-v \in \mathcal{A}^{p, q}(\Omega)$. Therefore, if $q<n$ or $q=n=2$, then $u=v$ and $u \in W_{0}^{1, q}(\Omega)$. Otherwise, $u=v+\lambda \in W_{0}^{1, q}(\Omega)$ with $\lambda \in \mathcal{A}^{p, q}(\Omega)$.

We complete this Note by an similar result for the three-dimensional Oseen equations with an analogous proof.

Theorem 2.3. Let $1<p<q<\infty$ and $\Omega \subset \mathbb{R}^{3}$ be an exterior domain with $C^{1,1}$ boundary.

i) If $q<4$, then $\mathcal{N}^{p, q}(\Omega)=\{(\mathbf{0}, 0)\}$.

ii) If $q \geq 4$, then

$$
\mathcal{N}^{p, q}(\Omega)=\left\{\left(\boldsymbol{\lambda}_{c}-\boldsymbol{c}, \mu_{c}\right) ; \boldsymbol{c} \in \mathbb{R}^{3}\right\}
$$

where $\left(\boldsymbol{\lambda}_{c}, \mu_{c}\right)$ is the unique solution of the following system

$$
-\Delta \boldsymbol{\lambda}_{c}+\frac{\partial \boldsymbol{\lambda}_{c}}{\partial x_{1}}+\nabla \mu_{c}=0, \operatorname{div} \boldsymbol{\lambda}_{c}=0 \text { in } \Omega, \boldsymbol{\lambda}_{c}=\boldsymbol{c} \text { on } \Gamma,
$$

such that $\boldsymbol{\lambda}_{c} \in \bigcap_{r>4 / 3} \mathbf{X}_{0}^{1, r}(\Omega)$ and $\mu_{c} \in \bigcap_{r>3 / 2} L^{r}(\Omega)$. Moreover, we have $\boldsymbol{\lambda}_{c} \in$ $\mathbf{L}^{s}(\Omega) \cap \mathbf{L}^{\infty}(\Omega)$ for all $s>2$.

Here, the kernel $\mathcal{N}^{p, q}(\Omega)$ of the exterior Oseen system is defined by

$$
\begin{aligned}
\mathcal{N}^{p, q}(\Omega)= & \left\{(\boldsymbol{u}, \pi) \in\left[\mathbf{X}_{0}^{1, p}(\Omega)+\mathbf{X}_{0}^{1, q}(\Omega)\right] \times\left[L^{p}(\Omega)+L^{q}(\Omega)\right]\right. \\
& \mathbf{T}(\boldsymbol{u}, \pi)=(\mathbf{0}, 0) \text { in } \Omega, \boldsymbol{u}=0 \text { on } \Gamma\}
\end{aligned}
$$

with $1<p<q<\infty$.

\section{References}

[1] R. A. Adams, Sobolev Spaces, Academic Press, New York, (2003).

[2] C. Amrouche, V. Girault, J. Giroire, Weighted Sobolev spaces for the Laplace equation in $\mathbb{R}^{n}$, Journal de Mathématiques Pures et Appliquées, 73 (6), 579-606, (1994).

[3] C. Amrouche, V. Girault, J. Giroire, Dirichlet and Neumann exterior problems for the $n$-dimensional Laplace operator: an approach in weighted Sobolev spaces, Journal de Mathématiques Pures et Appliquées, 76 (1), 55-81, (1997). 